\title{
Comparison of a minimally invasive surgical technique with open surgery for transverse patella fractures
}

\author{
JIE SHAO $^{1 *}$, JIABAO WANG $^{2 *}$, YU CHEN $^{1}$, NINGFANG MAO $^{1}$, QIULIN ZHANG $^{1}$ and HAIJIAN NI ${ }^{1}$ \\ ${ }^{1}$ Department of Orthopedics, Changhai Hospital, The Second Military Medical University, Shanghai 200433; \\ ${ }^{2}$ Department of Orthopedics, Suzhou First People's Hospital, Suzhou, Anhui 234000, P.R. China
}

Received November 29, 2017; Accepted October 26, 2018

DOI: $10.3892 /$ etm.2019.8078

\begin{abstract}
The current study aimed to compare a minimally invasive surgical technique with open surgery for the treatment of transverse patella fractures. A total of 38 patients with displaced transverse fractures of the patella were included in the current prospective study. A total 21 of them were treated with a minimally invasive surgical (MIS) technique and the other 17 patients had open surgery (OS) when using the Cable Pin System. Postoperative comparisons were based on radiographs, the visual analog scale (VAS) of pain, their ranges of motion and the Bostman grading scale. Compared with the OS group, the VAS score for pain was significantly lower and the angle of active flexion was better in the MIS group at 4 and 8 weeks. The angle of active extension at 4 weeks and the Bostman score at 8 weeks after surgery were better in the MIS group, although the differences became non-significant at 8 weeks and 12 months. In conclusion, the MIS technique for transverse patella fractures provided better clinical results and the knees functioned with less pain when compared with the OS group in the early period following surgery. MIS could be an adequate alternative in the treatment of transverse patella fractures.
\end{abstract}

\section{Introduction}

Patellar fractures comprise $0.5-1.5 \%$ of all types of bone fractures (1). Transverse fractures are the most common type of patella fracture, which often cause a functional disability of the knee extensor mechanism (2). Surgical treatment is recommended when there is $>3 \mathrm{~mm}$ of fragment separation

Correspondence to: Dr Qiulin Zhang or Dr Haijian Ni, Department of Orthopedics, Changhai Hospital, The Second Military Medical University, 168 Changhai Road, Shanghai 200433, P.R. China

E-mail: zhangqiulin@189.cn

E-mail: nihaijianch@163.com

${ }^{*}$ Contributed equally

Key words: patella, transverse fracture, minimally invasive surgery, open surgery, internal fixation or a step-like discontinuity on the articular surface $>2 \mathrm{~mm}$ (3). The objectives of surgical treatment include anatomical reduction of the articular surface, rigid internal fixation, and the reconstruction of the knee extensor mechanism, facilitating earlier functional exercises of the knee joint and avoiding post-traumatic patellofemoral arthritis (4-6).

Currently, methods combining interfragmentary screw fixation with the tension band principle have been demonstrated to provide enhanced fixation strength when compared with the modified tension band construct alone, and have been more and more widely used for the treatment of transverse patella fractures (7-10). However, most of these methods are still performed with an open technique that requires a long skin incision with a substantial soft tissues dissection, resulting in a higher probability of postoperative adhesions, prolonged disability (resulting in time off work) and cosmetically unsightly scars (11).

In clinical practice, the authors of the current study developed a new minimally invasive surgical (MIS) technique to be used instead of the traditional open surgery (OS) technique when using the Zimmer ${ }^{\circledR}$ Cable-Ready ${ }^{\circledR}$ Cable Pin System, a combination of the interfragmentary screws (paralleled pins) and the tension band wire (in a 'figure eight' formation) for fixation of transverse patella fractures. The objective of the current prospective study was to compare the MIS technique with OS by analyzing postoperative clinical and radiographic results.

\section{Patients and methods}

Patient groups and randomization. Between April 2014 and July 2015, 38 patients with clinical and X-ray evidence of transverse patella fractures with displacements $>3 \mathrm{~mm}$ were recruited at the Department of Orthopaedics, Changhai Hospital Affiliated to the Second Military Medical University (Shanghai, China). Age and sex distributions are presented in Table I. Among them, 21 patients were randomly assigned (by a coin flip) to the MIS group while 17 patients were assigned to the OS group by a scientist who was blinded to the present study. Exclusion criteria included an open fracture, a lower limb fracture on the same side, chronic degenerative joint disease, previous knee surgical intervention, peripheral neural damage and osteoporosis defined as bone mineral density value 2.5 standard deviations or more below peak bone mass 
(T-score $\leq-2.5$ ) measured by dual-energy X-ray absorptiometry (12). The current study was approved by the ethics review committee of the Second Military Medical University and written informed consent was obtained from all patients.

The Cable Pin System. The Zimmer ${ }^{\circledR}$ Cable-Ready ${ }^{\circledR}$ Cable Pin System (Zimmer Biomet Holdings, Inc., Warsaw, IN, USA) is a new type of internal fixation system, which consists of a cable and a pin (Fig. 1). One end of the system is the pin, a partially threaded $4.0 \mathrm{~mm}$ cancellous screw, which can provide compression between fragments and prevent the cancellous screw from backing out. The other end of the system is a guide needle, which can easily be passed through bone tunnels. The material connecting the two ends is a cable with a special braiding structure. It is braided from 19 wires with each wire containing 7 monofilament wires with a diameter of $0.005 \mathrm{~mm}$. Therefore, a cable comprises 133 monofilament wires. This design provides the cable with great strength and excellent pliability.

Surgical technique. All surgeries were performed by the same surgeon (QZ). Spinal anesthesia or combined spinal-epidural anesthesia was adopted for the surgery. The patient was positioned in the supine position, with a sterile soft cushion used to support the injured knee, leaving the joint in a $20^{\circ}$ flexed position, allowing the knee to perform flexion-extension movements.

i) MIS surgical technique. If the minor transverse fracture fragment was at the proximal end and the major fragment was at the distal end of the patella (Fig. 2), the site of the proximal fragment would be confirmed and a $\sim 3 \mathrm{~cm}$ longitudinal midline incision would be made from the proximal end of the patella down to the lower side of the proximal fragment (Fig. 3). The incision was from the skin to the prepatellar fascia. The soft tissues were pulled downwardto expose the fracture line. Any hematomas and traumatic bone debris were removed, and the articular cavity was washed with normal saline solution. Manipulative reduction was made with the assistance of two towel clamps applied percutaneously. During the reduction procedure, the knee joint was flexed and extended several times, and the fragments were squeezed and pressed against to the femoral condyle to facilitate the reduction of the articular surface. A K-wire was then drilled superiorly and percutaneously into the patella from the lower side of the apex for temporary fixation following the reduction (Fig. 4A). A C-arm fluoroscopy was used to confirm the anatomical reduction of the articular surface (Fig. 4B), and then the prepatellar fascia was sutured.

The proximal $3 \mathrm{~cm}$ incision was then pulled upward and the inserting points for the two pins were selected at the medial and lateral one-third of the superior pole of the patella. Using a drill guide to protect the quadriceps tendon, the pins of the Cable Pin System were inserted into the patella in an inferior direction parallel to the articular surface. The threaded portions of the pins were drilled across the fracture site, and the two pins were directed in the patella parallel to each other and perpendicular to the fracture line, which would provide compression between the fragments (Fig. 5). Each pin was drilled until it was slightly
Table I. Patient characteristics.

\begin{tabular}{lccc}
\hline Characteristic & $\begin{array}{c}\text { MIS } \\
\mathrm{n}=21\end{array}$ & $\begin{array}{c}\text { OS } \\
\mathrm{n}=17\end{array}$ & P-value \\
\hline Sex & & & \\
Male & 14 & 11 & $0.899^{\mathrm{a}}$ \\
Female & 7 & 6 & \\
Age (years) & $42.2 \pm 12.4$ & $40.3 \pm 10.5$ & $0.587^{\mathrm{b}}$ \\
19-39 & 8 & 8 & $0.821^{\mathrm{a}}$ \\
821 & 13 & 9 & \\
Side of injury & & & \\
Left & 12 & 6 & $0.310^{\mathrm{a}}$ \\
Right & 9 & 11 & \\
Mechanism of injury & & & \\
Fall & 14 & 15 & $0.148^{\mathrm{c}}$ \\
Traffic accident & 5 & 2 & \\
SRT & 2 & 0 & \\
TLIO (hours) & $33.6 \pm 18.4$ & $38.3 \pm 20.2$ & $0.418^{\mathrm{b}}$ \\
\hline
\end{tabular}

All values are mean \pm standard deviation or number of patients.

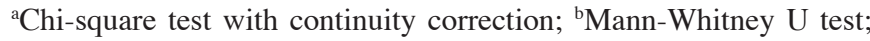
${ }^{\mathrm{c}}$ Fisher exact test (fall versus all others). MIS, minimally invasive surgery; OS, open surgery; SRT, sports-related trauma; TLIO, time lag between injury and operation.

countersunk into the bone cortex to prevent protrusion of the pin into the soft tissues. A $5 \mathrm{~mm}$ incision was made at the medial and lateral side of the distal one-fourth to one-fifth of the patella, respectively. Following this, two subcutaneous tunnels, starting from the proximal $3 \mathrm{~cm}$ incision and ending at each of the distal $5 \mathrm{~mm}$ incision, were created using a pair of curved forceps to divide the tissues under the deep fascia covering the distal fracture fragment of the patella. A horizontally directed bone tunnel was created anterior to the pins, in the distal fragment, using a drill bit through the medial and lateral $5 \mathrm{~mm}$ incisions (Fig. 6).

The leader of one cable was passed through the prepatellar subcutaneous tunnel from the proximal $3 \mathrm{~cm}$ incision (assumed as incision $\mathrm{X}$ ) to the diagonal distal $5 \mathrm{~mm}$ incision (assumed as incision Y; Fig. 7A). The cable was then inserted into the horizontally directed bone tunnel from incision $\mathrm{Y}$ and out of the tunnel at the other distal $5 \mathrm{~mm}$ incision (assumed as incision Z) (Fig. 7B). Finally, the same cable was passed through the other prepatellar subcutaneous tunnel from incision $\mathrm{Z}$ to $\mathrm{X}$ (Fig. 7C). The Cable Pin Crimper (Zimmer Biomet Holdings, Inc.) was used to pull the two cables crossed at incision $X$ to remove slack and fasten them in a crimp, forming a 'figure eight' (Fig. 7D). A cable cutter was used to cut the excess cables flush at the crimp (Fig. 8). Thus, with the exception of the interfragmentary screw fixation by the two paralleled pins, the cable was in an incomplete 'figure eight', which reflected the tension band principle (Fig. 9). If the fracture line was in the distal half of the patella, the midline longitudinal incision would be made at the distal end of the patella, accordingly, and the two pins would be reversed and directed superiorly from the lower side. 


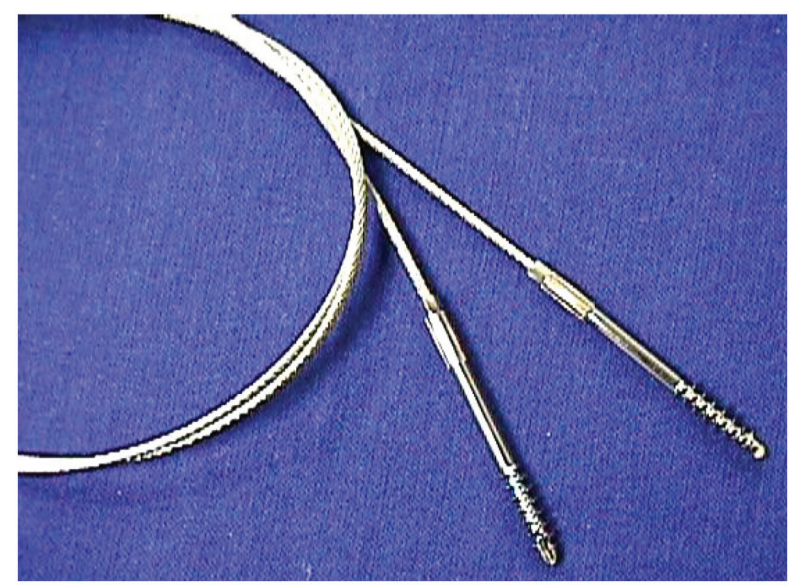

Figure 1. Two sets of the Cable Pin System. Each setconsists of a cable and a pin (a partially threaded $4.0 \mathrm{~mm}$ cancellous screw).

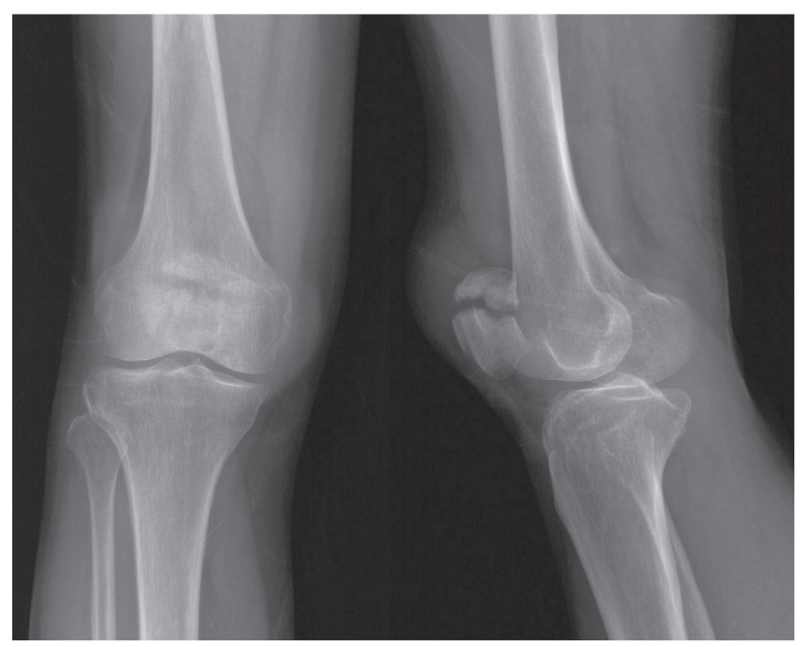

Figure 2. Preoperative radiographs. The radiographsdemonstrated the displaced transverse fracture of the patella. The fracture line was near the proximal one-third of the patella.

ii) Open surgery. The conventional midline longitudinal incision over the patella, which was $\sim 12 \mathrm{~cm}$ in length, was made and the fractures exposed as described previously (13). Following the preliminary reduction of the fracture, two pins of the Cable Pin System were inserted in parallel into the patella through the midline longitudinal incision to achieve interfragmentary compression. The two cables were crossed in a 'figure eight' over the anterior surface of the patella, with the leader of one cable passed through the horizontally directed bone tunnel, and were pulled to remove slack and fastened in a crimp. The excess cable flush was cut at the crimp.

Postoperative treatments. For all the 38 patients, an elastic bandage was used for 2 days after surgery to reduce swelling of the knee and the likelihood of a hematoma. No immobilization was recommended. Passive exercise was initiated 1 day after the surgery by a continuous passive motion machine (Kinetec Optima ${ }^{\circledR}$; Kinetec S.A.), for three, 1-h sessions, starting from 0 to $60^{\circ}$, increasing $15^{\circ}$ per day until $90^{\circ}$ was achieved. This training lasted for 3-5 days. Active flexion exercises of the knee joint in prone position were encouraged.

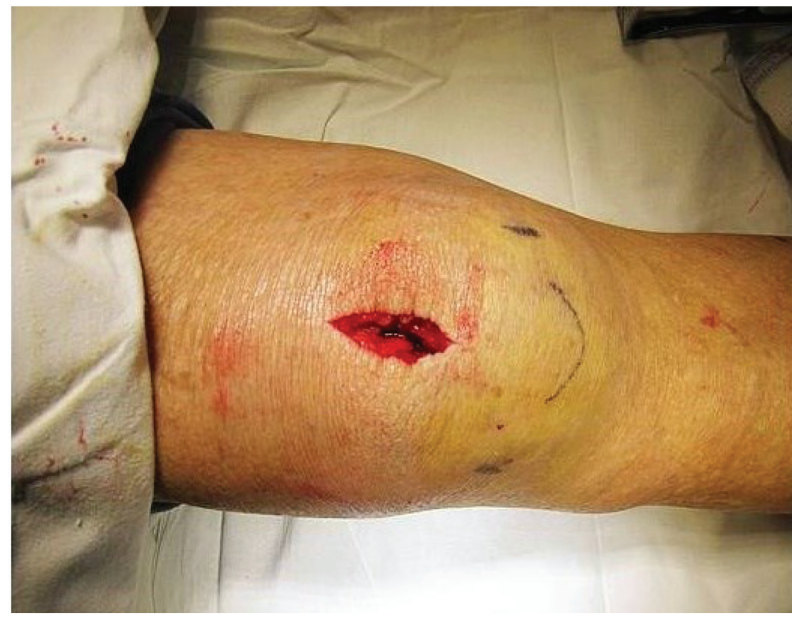

Figure 3. Incision at the longitudinal midline. The longitudinal midline incision was made from the proximal end of the patella down to the lower side of the proximal fragment.
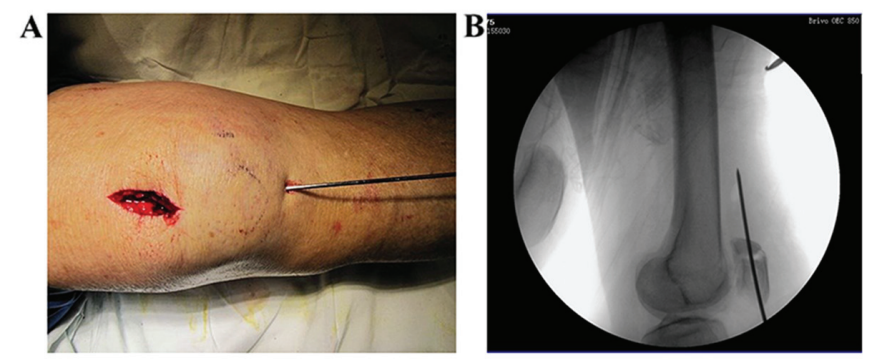

Figure 4. Fracture reduction and temporary fixation. (A) A K-wire for temporary fixation following the reduction. (B) A C-arm fluoroscopy was used to confirm the anatomical reduction of the articular surface.

The patients began to get out of bed 3 days after surgery and were encouraged to start bearing weight on the leg that was operated on during level walking. Active extension exercises of the knee joint were allowed 3 weeks after surgery, and full weightbearing was allowed after the fracture was healed following a radiographic assessment.

Postoperative follow-up and assessment. Clinical and radiographical follow-up were performed at 4 and 8 weeks, and at 12 months postoperatively. All the x-ray films were read independently by two well-trained orthopedic surgeons (QZ and $\mathrm{HN}$ ). At 4 and 8 weeks, pain was measured using the visual analog scale (VAS) (14) with scores between 0 (no pain) and 10 (the most intense pain), and active flexion and extension of the knee joint were measured in degrees by a goniometry. The function of the knee was evaluated by the clinical grading scale, defined by Bostman et al (15), at week 8 and month 12 . The total score was stratified as follows: $<20$, unsatisfactory; 20-27, good; 28-30, excellent.

Statistical analysis. All values are mean \pm standard deviation or number of patients. The Mann-Whitney $U$ test was used to compare quantitative variables and the chi-square test with continuity correction or the Fisher exact test was used to compare the frequency distributions of categorical variables between the two groups. All statistical analyses 


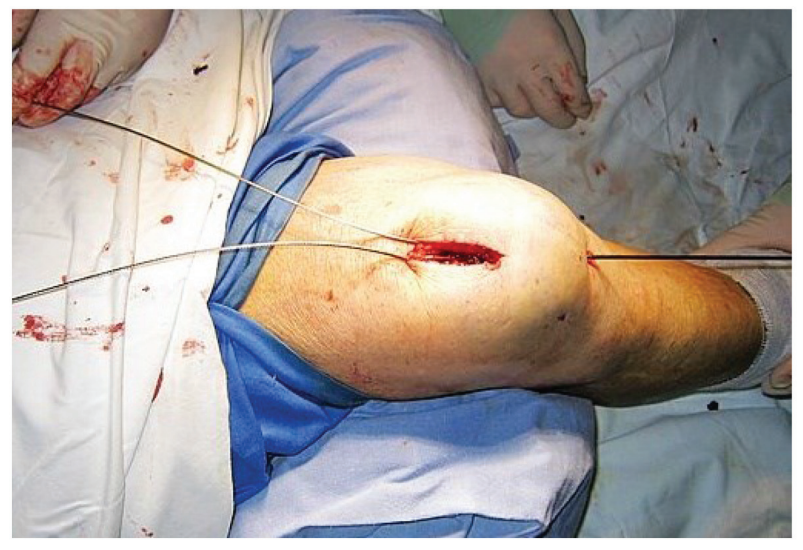

Figure 5. Pin insertion. The pins of the Cable Pin System were inserted into the patella with the threaded portions drilled across the fracture site, providing compression between the fragments.

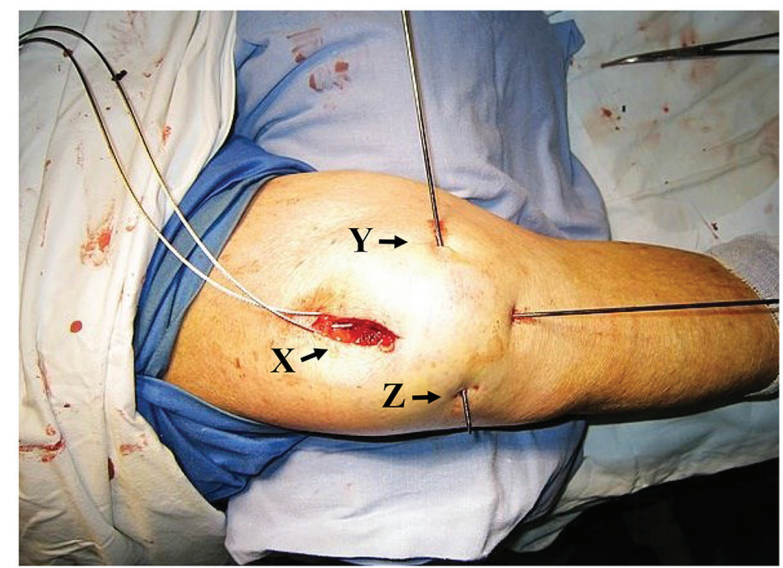

Figure 6. Subcutaneous and bone tunnels. A small $5 \mathrm{~mm}$ incision was made at the median and lateral side of the distal one-fourth to one-fifth of the patella, respectively. Two subcutaneous tunnels (from $\mathrm{X}$ to $\mathrm{Y}$ and from $\mathrm{X}$ to Z) were created. A horizontally directed bone tunnel was created in the distal fragment through the median and lateral $5 \mathrm{~mm}$ incisions. $\mathrm{X}$, the longitudinal midline incision from the proximal end of the patella down to the lower side of the proximal fragment; $Y$, the incision at the median side of the distal one-fourth of the patella; $\mathrm{Z}$, the incision at the lateral side of the dista one-fifth of the patella.

were performed using SPSS 15.0 statistical software (SPSS Inc., Chicago, IL, USA). $\mathrm{P}<0.05$ indicated that the difference between groups was statistically significant.

\section{Results}

All patients in the two groups completed $\geq 1$ year of follow-up time. No statistically significant differences were identified in sex and age distributions, side distribution of the fracture, the mechanism of injury or the time lag between injury and operation between the two groups (Table I).

The average operating time was 49.0 and $52.2 \mathrm{~min}$ in the MIS group and the OS group, respectively, and no significant difference was observed between the two groups (Table II). The VAS score for pain was significantly greater in the OS group compared with the MIS group during the active movement of the injured knee joint at 4 and 8 weeks after surgery. The average
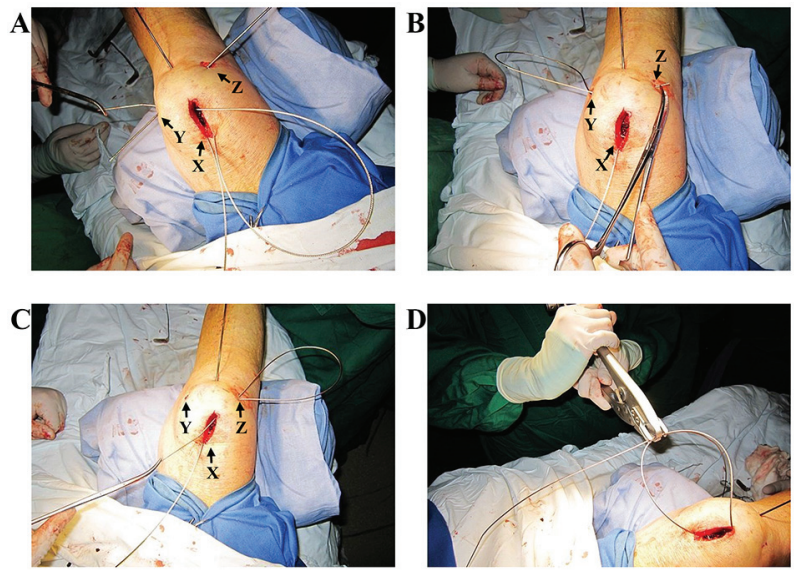

Figure 7. Paths of the cables used. (A) The leader of one cable was passed through the prepatellar subcutaneous tunnel from $X$ to $Y,(B)$ through the horizontally directed bone tunnel from $\mathrm{Y}$ to $\mathrm{Z}$ (C) finally back to X. (D) A crimper was used to pull the two cables and cross then at $X$ to remove slack and fasten them in a crimp. $\mathrm{X}$, the longitudinal midline incision from the proximal end of the patella down to the lower side of the proximal fragment; $\mathrm{Y}$, the incision at the median side of the distal one-fourth of the patella; $\mathrm{Z}$, the incision at the lateral side of the distal one-fifth of the patella.
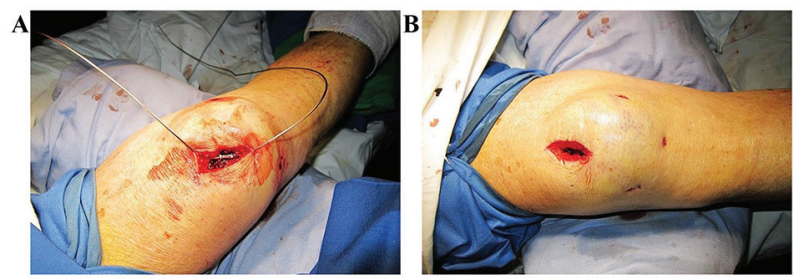

Figure 8. Cable crimping and cutting. (A) The two cables were pulled and crossed at the longitudinal midline incision and fastened in a crimp. (B) A cable cutter was used to cut the excess cables flush at the crimp.
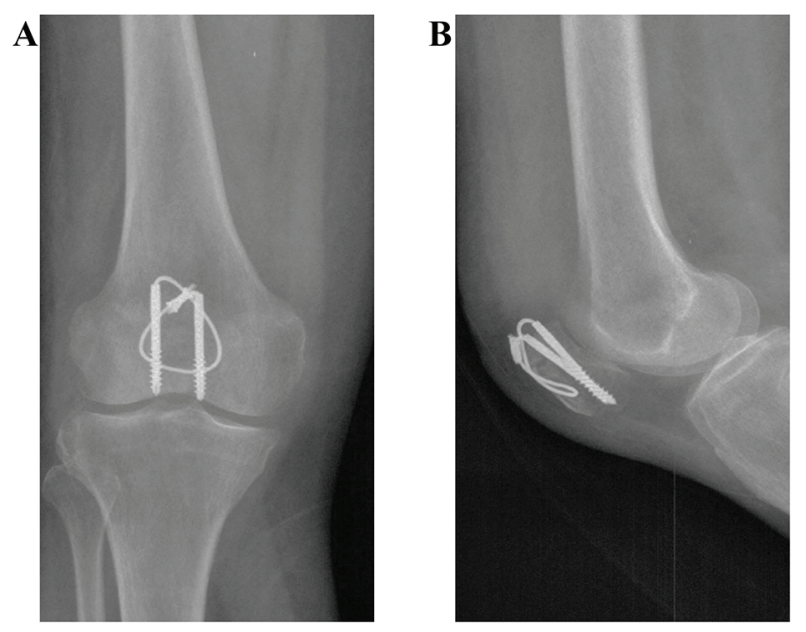

Figure 9. Postoperative radiographs 8 weeks after surgery. (A) A postoperative anteroposterior radiograph. (B) A postoperative lateral radiograph demonstrating the anatomic reduction of the patella fracture.

angle of active extension of the injured knee was significantly better in the MIS group at 4 weeks after surgery, but this difference became non-significant at 8 weeks, when compared with the OS group. The average angle of active flexion was significantlybetter in the MIS group at 4 and 8 weeks compared with 
Table II. Comparison of postoperative results in the MIS and OS patient groups.

\begin{tabular}{lccc}
\hline Result & $\begin{array}{c}\text { MIS } \\
\mathrm{n}=21\end{array}$ & $\begin{array}{c}\text { OS } \\
\mathrm{n}=17\end{array}$ & P-value \\
\hline $\begin{array}{l}\text { Operating time (min) } \\
\text { VAS score for pain }\end{array}$ & $49.0 \pm 10.2$ & $52.2 \pm 12.7$ & 0.461 \\
4 weeks & $4.0 \pm 1.1$ & $5.4 \pm 1.0$ & 0.001 \\
8 weeks & $1.6 \pm 1.3$ & $3.5 \pm 1.6$ & 0.001 \\
Flexion $\left(^{\circ}\right)$ & & & \\
4 weeks & $52.6 \pm 18.3$ & $35.5 \pm 10.6$ & 0.004 \\
8 weeks & $91.1 \pm 14.3$ & $75.3 \pm 15.2$ & 0.005 \\
Extension $\left(^{\circ}\right)$ & & & \\
4 weeks & $-5.0 \pm 4.7$ & $-9.4 \pm 6.6$ & 0.033 \\
8 weeks & $-1.2 \pm 4.4$ & $-3.5 \pm 5.5$ & 0.184 \\
Bostman score & & & \\
8 weeks & $28.2 \pm 1.7$ & $27.1 \pm 2.2$ & 0.038 \\
12 months & $28.9 \pm 1.1$ & $28.4 \pm 1.1$ & 0.162 \\
\hline
\end{tabular}

All values are mean \pm standard deviation. The Mann-Whitney $U$ test wasused to compare the groups. MIS, minimally invasive surgery; OS, open surgery; VAS, visual analog scale.

the OS group. The mean Bostman score reached 28.2 (range, 25-30) in the MIS group 8 weeks after surgery, indicating asignificantly better function of the injured knee compared with the OS group, though this difference became non-significant at 12 months. Until the last follow-up visit, no defect of the articular surface, patellofemoral arthritic changes, breaking or backing out of pin, or cable rupture was detected through radiological examinations. The longitudinal midline incision was only $\leq 3 \mathrm{~cm}$ in the MIS group compared with an extensive soft tissue dissections in the OS group (Fig. 10).

\section{Discussion}

MIS is the trend of development in modern surgery (16). This less painful technique has been demonstrated to be associated with earlier mobilization and rehabilitation, a higher functional score of the knee, fewer joint adhesions or less ankylosis, and lower incidence of wound complications (16-20). Several studies have investigated MIS or the percutaneous technique for transverse patella fractures (17-19). Luna-Pizarro et al (17) performed percutaneous patellar osteosynthesis using tension band wiring with the aid of a specifically designed device and revealed that it was superior to the conventional OS approach. However, it was pointed out that the intraarticular hematoma and bone marrow particles or small chondral/osteochondral fragments could not be washed out of the joint, which may have caused joint adhesions and prolonged disability during the rehabilitation period (21). Other studies assessed the arthroscopic-assisted reduction and percutaneous fixation of displaced patellar fractures $(18,19)$. However, the technique was technically demanding and not suitable for the fractures accompanied by the disruption of the extensor mechanism $(18,19)$.

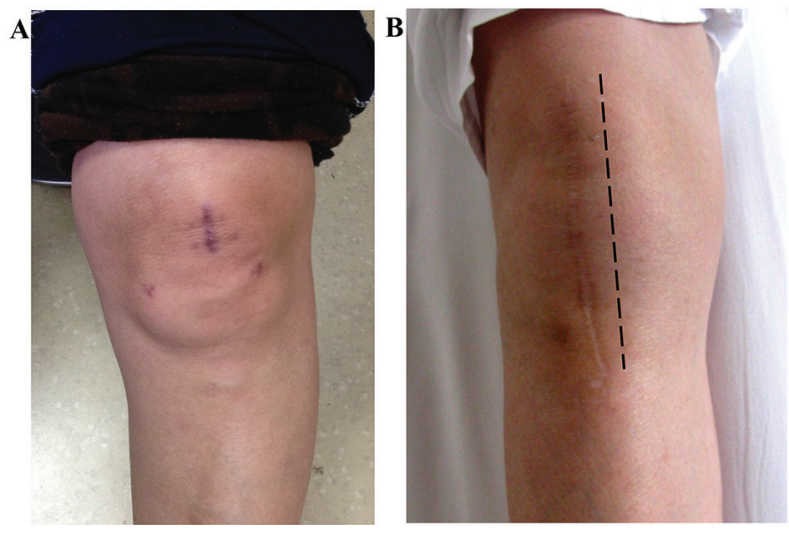

Figure 10. Postoperative appearances of the incisions 1 year after surgery. (A) Minimally invasive incisions in the MIS group. (B) Longitudinal midline incision in the OS group. MIS, minimally invasive surgery; OS, open surgery.

In the present study, a MIS technique was introduced and compared with OS when using the Cable Pin System for transverse patella fractures. The longitudinal midline incision was only $\leq 3 \mathrm{~cm}$, with two subcutaneous tunnels and a bone tunnel, instead of extensive soft tissue dissections. In the MIS group, a lower VAS score for pain was observed at 4 and 8 weeks after surgery, which could be partially attributed to less destruction of the soft tissues compared with the OS group. Additionally, the mean angle of active flexion or extension of the injured knee and the Bostman functional score, were greater in the MIS group at 4 weeks, and the mean angle of active flexion was still greater in the MIS group at 8 weeks compared with the OS group, indicating better knee functions in the early period after surgery. Although those differences between the two groups could no longer be identified at the final follow-up, the MIS technique may help patients get back to the daily work and life earlier with less pain and smaller scars, improving patient satisfaction.

The authors of the current study elected to not use a completely percutaneous technique but rather a MIS or 'semi-percutaneous' technique as they hypothesized that, unlike shaft fractures of the limbs, the transverse patella fracture was more often an intraarticular fracture. Intraarticular hematomas and traumatic debris, which may cause joint adhesions and prolonged disability during the rehabilitation period, should be washed out of the joint. Through the minimally invasive incision, all the procedures, including removal of intraarticular hematomas and traumatic debris, dislodgment of the entrapped soft tissue, the reduction of the fractures and reconstruction of the knee extensor mechanism, could be carried out. The MIS technique introduced in the current study had minimal soft tissue disruption, fewer complications (data not shown) and better outcomes. MIS techniques may also be applicable to other patterns of internal fixation, such as interfragmentary screws with tension band wiring or cable.

A limitation of this MIS technique was that the reduction would be difficult through the minimally invasive incision when there were multiple fragments at the fracture site and thus it was not suited to those patella fractures with comminution of the fragments. Additionally, it is possible that there were complications, which we had not identified due to the short follow-up time, and longer follow-ups are required in 
the future. Another limitation was the sample size and small differences of in results between the two groups did not have clinical relevance.

In conclusion, the MIS technique for transverse patella fractures was demonstrated to provide better clinical results and knee functions with less pain when compared to OS in the early period following surgery. It could be an adequate alternative in the treatment of transverse patella fractures. Large-scale studies are necessary to further confirm the effectiveness of this technique.

\section{Acknowledgements}

Not applicable.

\section{Funding}

No funding was received.

\section{Availability of data and materials}

The datasets used and/or analyzed during the current study are available from the corresponding author on reasonable request.

\section{Authors' contributions}

JS, JW, YC and NM performed the experiments. QZ and HN designed and directed the experiments. JS and JW wrote the manuscript.

\section{Ethics approval and consent to participate}

The current study was approved by the ethics review committee of the Second Military Medical University and written informed consent was obtained from all patients.

\section{Patient consent for publication}

All patients consented to the publication of the current article.

\section{Competing interests}

The authors declare that they have no competing interests.

\section{References}

1. Galla M and Lobenhoffer P: Patella fractures. Chirurg 76: 987-997, 2005 (In German).

2. Boström A: Fracture of the patella. A study of 422 patellar fractures. Acta Orthop Scand Suppl 143: 1-80, 1972.
3. Gosal HS, Singh P and Field RE: Clinical experience of patellar fracture fixation using metal wire or non-absorbable polyester - a study of 37 cases. Injury 32: 129-135, 2001.

4. Weber MJ, Janecki CJ, McLeod P, Nelson CL and Thompson JA: Efficacy of various forms of fixation of transverse fractures of the patella. J Bone Joint Surg Am 62: 215-220, 1980.

5. Lotke PA and Ecker ML: Transverse fractures of the patella. Clin Orthop Relat Res: 180-184, 1981

6. Catalano JB, Iannacone WM, Marczyk S, Dalsey RM, Deutsch LS, Born CT and Delong WG: Open fractures of the patella: Long-term functional outcome. J Trauma 39: 439-444, 1995.

7. Burvant JG, Thomas KA, Alexander R and Harris MB: Evaluation of methods of internal fixation of transverse patella fractures: A biomechanical study. J Orthop Trauma 8: 147-153, 1994.

8. Cekin T, Tükenmez M and Tezeren G: Comparison of three fixation methods in transverse fractures of the patella in a calf model. Acta Orthop Traumatol Turc 40: 248-251, 2006 (In Turkish).

9. Curtis MJ: Internal fixation for fractures of the patella. A comparison of two methods. J Bone Joint Surg Br 72: 280-282, 1990.

10. Cho JH: Percutaneous cannulated screws with tension band wiring technique in patella fractures. Knee Surg Relat Res 25: 215-219, 2013.

11. Gardner MJ, Griffith MH, Lawrence BD and Lorich DG: Complete exposure of the articular surface for fixation of patellar fractures. J Orthop Trauma 19: 118-123, 2005.

12. Kanis JA, Melton LJ 3rd, Christiansen C, Johnston CC and Khaltaev N: The diagnosis of osteoporosis. J Bone Miner Res 9: 1137-1141, 1994.

13. Sanders R and Gregory PR: Patella fractures and extensor mechanism injuries. In: Skeletal trauma basic science, management, and reconstruction. 4th edition. Saunders Elsevier Inc., Philadelphia, PA, pp2013-2015, 2009.

14. Butler PV: Linear analogue self-assessment and procrustean measurement: A critical review of visual analogue scaling in pain assessment. J Clin Psychol Med Settings 4: 111-129, 1997.

15. Bostman O, Kiviluoto O and Nirhamo J: Comminuted displaced fractures of the patella. Injury 13: 196-202, 1981.

16. Zeng BF: Minimally invasive surgery in fracture management. Chin Med J (Engl) 121: 1349-1351, 2008.

17. Luna-Pizarro D, Amato D, Arellano F, Hernandez A and Lopez-Rojas P: Comparison of a technique using a new percutaneous osteosynthesis device with conventional open surgery for displaced patella fractures in a randomized controlled trial. J Orthop Trauma 20: 529-535, 2006.

18. El-Sayed AM and Ragab RK: Arthroscopic-assisted reduction and stabilization of transverse fractures of the patella. Knee 16: 54-57, 2009.

19. Turgut A, Günal I, Acar S, Seber S and Göktürk E: Arthroscopic assisted percutaneous stabilization of patellar fractures. Clin Orthop Relat Res: 57-61, 2001.

20. Atesok K, Doral MN, Whipple T, Mann G, Mei-Dan O, Atay OA, Beer Y, Lowe J, Soudry M and Schemitsch: Arthroscopy-assisted fracture fixation. Knee Surg Sports Traumatol Arthrosc 19: 320-329, 2011.

21. Kose KC, Kuru I, Maralcan G and Altinel L: Comparison of a technique using a new percutaneous osteosynthesis device with conventional open surgery for displaced patella fractures. J Orthop Trauma 21: 77-78, 2007. International (CC BY-NC-ND 4.0) License. 